\title{
Effects of Various Foods Intakes on Plasma Levels of Trans Fatty Acids in Japanese Old Men
}

\author{
F. Shimizu' ${ }^{1}$ Y. Ishii ${ }^{1}$, M. Ogawa ${ }^{1}$, T. Takao ${ }^{1}$, S. Koba ${ }^{2}$, A. Takada ${ }^{3 *}$ \\ ${ }^{1}$ Faculty of Life and Environmental Sciences, Showa Women's University, Tokyo, Japan \\ ${ }^{2}$ Division of Cardiology, Department of Medicine, Showa University School of Medicine, Tokyo, Japan \\ ${ }^{3}$ International Projects on Food and Health (NPO), Tokyo, Japan \\ Email: *takadaa@mwd.biglobe.ne.jp
}

How to cite this paper: Shimizu, F., Ishii, Y., Ogawa, M., Takao, T., Koba, S. and Takada, A. (2018) Effects of Various Foods Intakes on Plasma Levels of Trans Fatty Acids in Japanese Old Men. Food and Nutrition Sciences, 9, 797-805.

https://doi.org/10.4236/fns.2018.97059

Received: June 19, 2018

Accepted: July 8, 2018

Published: July 11, 2018

Copyright (C) 2018 by authors and Scientific Research Publishing Inc. This work is licensed under the Creative Commons Attribution International License (CC BY 4.0).

http://creativecommons.org/licenses/by/4.0/

Open Access

\begin{abstract}
Background: Trans fatty acids are said to be formed by the partial hydrogenation of vegetable oils. Some amounts are produced in digestive organs of ruminants and present in dairy products or meat. In Japan, use of trans fatty acids in the foods is prohibited, thus trans fatty acids must come from foods or microbes in the digestive organs. Methods: Plasma levels of fatty acids including trans forms of healthy old men are measured by gas chromatography and correlations between various foods intakes and plasma levels of trans fatty acids such as palmitoelaidic, elaidic and linoelaidic acids are examined. Results: No correlations between various foods intakes and trans fatty acids were found except between intake of preference drinks such as tea or coffee and plasma levels of palmitoelaidic and linoelaidic acids. Conclusion: Since palmitoelaidic acid is cardioprotective, increase in plasma levels of palmitoelaidic acid may indicate that intakes of tea and coffee may be beneficial for heath by increasing palmitoelaidic acids.
\end{abstract}

\section{Keywords}

Fatty Acid, Trans Fatty Acid, Palmitoelaidic Acid, Elaidic Acid, Linoelaidic Acid, Saturated Fatty Acid, Unsaturated Fatty Acid, $\omega$ Fatty Acid, DHA

(Docosahexanoic Acid), EPA (Eicosapentanoic Acid), AA (Arachidonic Acid)

\section{Introduction}

Trans fatty acids, unsaturated fatty acids with at least one double bond in the trans form, are formed during the partial hydrogenation of vegetable oils. This process changes vegetable oils to semisolid fats, which can be used in margarines and commercial cooking. The average consumption of industrially produced 
trans fatty acids in US is $2 \%$ to $3 \%$ of total calories consumed [1].

Higher intakes of industrially-produced trans fatty acids (IP-TFA) [2] and of saturated fatty acids (SFAs) are associated with increased risk for CHD (coronary heart disease) [3] [4], and higher intakes of both the $\omega 6(n-6)$ polyunsaturated fatty acids (PUFAs) and the $n-3$ PUFAs are associated with lower risk of CHD [5]. Since estimation of dietary intakes of FAs is challenging (out of date databases, relies on memory, poor estimation of portion sizes, etc.) many researchers have begun to measure plasma/blood levels of FAs as more objective biomarkers of exposure.

Naturally present trans fatty acids are found in fats of ruminants such as cattle and goats [6]. Since use of IP-TFA is prohibited in Japan; TFAs in plasma are considered to be intaken as foods such as meats and other dairy products.

The few studies investigating these naturally derived trans FAs have found no clear association with some CHD risk factors or incident disease, although the results have been inconsistent [7].

Any investigation of the effects of trans fatty acid in specific populations should begin with the estimation of trans fatty acid intake in that population. However, few data are available on individual intakes estimated using trans fatty acid food composition tables in Asian countries, including Japan, in part because nutrient databases cannot keep up with the rapid rate of re-formulation of food oils which markedly changes IP-TFA levels. Several estimates for Japanese populations have been reported, but their validity is questionable. Accordingly, measuring circulating in vivo fatty acid levels is believed to provide important information of roles of TFA in health and diseases.

We have reported that plasma levels of the major industrially-produced trans fatty acids (IP-TFAs; elaidic and linoelaidic acids) were far higher in American men, and levels of the potentially cardioprotective, primarily ruminant-derived trans fatty acid palmitoelaidic acid (POA) were higher in Japanese. [8].

In the present studies, we examined relationships between various foods intake and plasma levels of 3 important TRAs such as palmitoelaidic, elaidic, and linoelaidic acids.

\section{Materials and Methods}

\subsection{Participants}

In Japan, we recruited 44 male volunteers older than 50 who were friends and family members of the research team for this study. Exclusion criteria included the use of medications to treat diabetes, hyperlipidemia, hypertension and/or cardiovascular disease (CVD). Smokers were also excluded.

\subsection{Analyses of Fatty Acids Levels of Plasma Samples}

We asked 44 healthy men older than 50 and checked their health carefully. We examined their blood samples and recruited them if there were no health problems such as diabetes, hypertension or not serious diseases experienced in the 
past. They did not smoke in the past. We also excluded people who took drugs for dyslipidemia, hyperglycemia, or hypertension. We collected blood samples early morning. Participants were asked not to eat anything after 21:00 PM the previous evening. Plasma specimens were collected for assays of blood parameters. We obtained an informed consent prior to conducting the protocol which had been approved by the Ethical Committee of Showa Women's University and Saiseikai Shibuya Satellite Clinic.

Fatty acids levels were measured in plasma obtained from ethylenediamine tetraacetic acid anticoagulated blood samples. Samples were frozen at -80 degrees until analyzed at Omegaquant, LLC (Sioux Falls, SD, USA). After thawing, an aliquot of plasma was combined (1:40 parts) with the methylating mixture (boron trifluoride in methanol [14\%], toluene, and methanol [35/30/35 v/v]), shaken at $100^{\circ} \mathrm{C}$ for 45 minutes. After cooling, 40 parts of both hexane and distilled water were added. After briefly vortexing, the samples were spun to separate layers, and an aliquot of the hexane layer that contained the fatty acid methyl esters was analyzed by gas chromatography as previously described [9].

\subsection{Other Plasma Factors}

Plasma factors were measured after plasma was separated from blood. Ethylenediamine tetra acetic acid (EDTA) was used as an anticoagulant. Blood glucose levels were measured by a hexokinase UV method. Insulin was measured by the CLEIA (chemiluminescent immunoassay) method. We also measured glycemic indexes after giving glucose and sucrose to participants, so that we did not use $\mathrm{HbAlc}$ as a marker of glycemia.

Lipid and lipoprotein concentrations such as total cholesterol, HDL (high density lipoprotein cholesterol), LDL (low density lipoprotein) cholesterol, and TG (triglyceride) were determined using a Polychem Chemistry Analyzer (Polymedco Inc.). FFA (free fatty acid) and the concentrations of $\omega$ fatty acids such as arachidonic acid, DHA, and EPA were measured by a gas chromatography.

Remnant lipoproteins (RLPs) were isolated from the serum to an immunoaffinity mixed gel containing anti-apolipoprotein A1 and anti-apolipoprotein B100 monoclonal antibodies (Japan Immunoresearch Laboratories, Takasaki, Japan), and the cholesterol and triglyceride concentrations of the unbound fraction were measured as RLP cholesterol and RLP-triglyceride, respectively.

\subsection{Studies of Foods Intakes}

Participants were given self-administered diet history questionnaires and described answers on each item by recollection of diets they took (7 days dietary recall). We used a brief-type self-administered diet history questionnaire (BDHQ) by using which the Japanese Ministry of Health, Labour and Welfare reports National Nutrition Surveys. From these questionnaires, we calculated the intakes of energy, and varieties of foods such as proteins, carbohydrates, lipids vitamins etc. 


\section{Results}

Table 1 shows that participants are average Japanese men over 50 years old.

Table 2 shows that plasma levels of various parameters of the present samples are within normal ranges of healthy Japanese old man [7].

Table 3 also shows plasma levels of various fatty acids including trans type such as plmitoelaidic, elaidic and linoelaidic acids.

Table 3 shows plasma levels of fatty acids including $\omega$ fatty acids and trans fatty acids. Compared with old men in USA, plasma levels of DHA and EPA were higher in Japanese men and plasma levels of elaidic and linoelaidic acids were higher in Old mane in USA [8].

Table 4 shows that there were no correlations between foods intakes and plasma levels of trans fatty acids and that only preference drinks such as tea and coffee had significant correlations with plasma levels of palmitoelaidic acid and linoelaidic acid.

\section{Discussion}

Most trans fats in the US diet are produced industrially during the partial

Table 1. Backgrounds of various parameters of healthy old men in Japan.

\begin{tabular}{cc}
\hline & Old men $(\mathrm{n}=44)$ \\
\hline Age & $62.4 \pm 9.6$ \\
Height $(\mathrm{m})$ & $1.68 \pm 0.07$ \\
Weight $(\mathrm{kg})$ & $68.8 \pm 10.9$ \\
BMI & $24.3 \pm 3.2$
\end{tabular}

Mean \pm SD

Table 2. Plasma parameters.

\begin{tabular}{cc}
\hline Subjects & Plasma levels \\
TG: triglyceride $(\mathrm{mg} / \mathrm{dL})$ & old men, $\mathrm{n}=44$ \\
HDL-Chol.: HDL-cholesterol $(\mathrm{mg} / \mathrm{dL})$ & $126.4 \pm 81.3$ \\
LDL-Chol.: LDL-cholesterol $(\mathrm{mg} / \mathrm{dL})$ & $60.9 \pm 14.6$ \\
T-Chol.: total cholesterol $(\mathrm{mg} / \mathrm{dL})$ & $123.7 \pm 30.2$ \\
RLP-Chol.: remnant cholesterol $(\mathrm{mg} / \mathrm{dL})$ & $209.9 \pm 32.3$ \\
DHLA: dihydrolipoic Acid $(\mu \mathrm{g} / \mathrm{mL})$ & $7.6 \pm 9.6$ \\
AA: arachidonic acid $(\mu \mathrm{g} / \mathrm{mL})$ & $36.5 \pm 10.3$ \\
EPA: eicosapentanoic acid $(\mu \mathrm{g} / \mathrm{mL})$ & $36.5 \pm 10.3$ \\
DHA: docosahexanoic acid $(\mu \mathrm{g} / \mathrm{mL})$ & $87.1 \pm 46.7$ \\
EPA/AA ratio & $158.6 \pm 52.2$ \\
Blood glucose $(\mathrm{mg} / \mathrm{dL})$ & $0.42 \pm 0.21$
\end{tabular}

Mean \pm SD 
Table 3. Plasma levels of fatty acids.

\begin{tabular}{|c|c|}
\hline Fatty acid (\%) & Old men \\
\hline Myristic & $0.7 \pm 0.2$ \\
\hline Palmitic & $22.0 \pm 1.3$ \\
\hline Palmitoelaidic (trans) & $0.2 \pm 0.1$ \\
\hline Palmitoleic & $1.9 \pm 0.6$ \\
\hline Stearic & $7.0 \pm 0.7$ \\
\hline Elaidic (trans) & $0.1 \pm 0.01$ \\
\hline Oleic & $20.0 \pm 2.6$ \\
\hline Linoelaidic (trans) & $0.2 \pm 0.1$ \\
\hline LA_Linoleic & $26.3 \pm 4.0$ \\
\hline Arachidic & $1.3 \pm 0.04$ \\
\hline GLA_gamma_Linolenic & $0.3 \pm 0.1$ \\
\hline Eicosenoic & $0.2 \pm 0.04$ \\
\hline ALA_alpha_Linolenic & $0.7 \pm 0.2$ \\
\hline Eicosadienoic & $0.2 \pm 0.03$ \\
\hline Behenic & $0.15 \pm 0.02$ \\
\hline DGLA_Dihomo_g_linolenic & $1.0 \pm 0.2$ \\
\hline AA_Arachdonic & $6.0 \pm 1.1$ \\
\hline EPA_eicosapentaenoic & $2.5 \pm 1.3$ \\
\hline Lignoceric & $0.22 \pm 0.10$ \\
\hline Nervonic & $0.33 \pm 0.18$ \\
\hline Docosatetraenoic & $0.12 \pm 0.04$ \\
\hline Docosapentaenoic-n6 & $0.14 \pm 0.04$ \\
\hline DPA_Docosapentaenoic-n3 & $0.68 \pm 0.26$ \\
\hline DHA_Docosahexaenoic & $5.0 \pm 1.5$ \\
\hline
\end{tabular}

Mean \pm SD.

hydrogenation of vegetable oils. Smaller amounts are present in dairy products and in meat from cows, sheep, and other ruminants, produced by bacteria in their stomachs. The predominant trans-isomer in ruminants is vaccenic acid (18:1 cis-11), from which conjugated linolenic acid (another trans fatty acid) can be formed.

We found that IP-trans fatty acids were lower in Japan vs the US [8]. The reported intake of IP-TFA is $0 \%$ in Japan, again supporting the observed differences in biomarker levels. Circulating 18:2 trans fatty acids were shown to be most adversely associated with total mortality, mainly due to the increased risk of CVD [9]. It was also positively associated with total mortality and CHD. In a recent study from Germany, total trans fatty acids in erythrocyte membranes were shown to be inversely associated with mortality, but this was mainly driven 
Table 4. Correlations between foods intakes and plasma levels of fatty acids.

\begin{tabular}{|c|c|c|c|}
\hline & Palmitelaidic & Elaidic & Linoelaidic \\
\hline Energy & 0.319 & -0.135 & 0.181 \\
\hline Protein & 0.239 & 0.031 & 0.034 \\
\hline Animal protein & 0.233 & 0.135 & 0.034 \\
\hline Vegetable protein & 0.193 & -0.081 & 0.152 \\
\hline Lipid & 0.187 & 0.120 & 0.034 \\
\hline Animal lipid & 0.188 & 0.154 & 0.094 \\
\hline Vegetable lipid & 0.167 & 0.027 & -0.066 \\
\hline Carbohydrate & 0.188 & -0.197 & 0.138 \\
\hline $\mathrm{Na}$ & 0.128 & 0.033 & -0.073 \\
\hline $\mathrm{K}$ & 0.223 & -0.015 & 0.137 \\
\hline $\mathrm{Ca}$ & 0.099 & 0.263 & -0.021 \\
\hline $\mathrm{Mg}$ & 0.283 & -0.003 & 0.143 \\
\hline Phosphrus & 0.230 & 0.088 & 0.020 \\
\hline Iron & 0.193 & -0.120 & 0.112 \\
\hline Zing & 0.109 & 0.081 & 0.072 \\
\hline Cupper & 0.211 & -0.066 & 0.163 \\
\hline $\mathrm{Mn}$ & 0.422 & -0.059 & 0.113 \\
\hline Retinol & 0.307 & -0.195 & 0.054 \\
\hline$\beta$ carotein & -0.001 & -0.049 & -0.005 \\
\hline Vit D & 0.295 & -0.001 & -0.066 \\
\hline Tochopherol & 0.167 & -0.049 & -0.004 \\
\hline Vit $K$ & 0.098 & 0.014 & 0.083 \\
\hline Vit B1 & 0.095 & 0.119 & 0.098 \\
\hline Vit B2 & 0.246 & 0.169 & 0.135 \\
\hline Niacine & 0.320 & -0.012 & 0.081 \\
\hline Vit B6 & 0.239 & -0.036 & 0.129 \\
\hline Vit B12 & 0.353 & -0.090 & 0.049 \\
\hline Folic acid & 0.333 & -0.120 & 0.080 \\
\hline Panthothenic acid & 0.299 & 0.120 & 0.083 \\
\hline Vit C & 0.214 & -0.040 & 0.034 \\
\hline Saturated fatty acids & 0.209 & 0.144 & 0.112 \\
\hline Monovalent fatty acids & 0.156 & 0.126 & -0.004 \\
\hline Multivalent fatty acids & 0.179 & 0.024 & -0.062 \\
\hline Cholesterol & 0.221 & -0.025 & 0.125 \\
\hline Soluble dietary fiber & 0.133 & -0.080 & 0.155 \\
\hline Insoluble dietary fiber & 0.125 & -0.047 & 0.076 \\
\hline Total dietary fiber & 0.082 & 0.027 & 0.089 \\
\hline Salt & 0.128 & 0.033 & -0.073 \\
\hline Preference drinks & $0.586^{\star *}$ & -0.263 & $0.511^{\star}$ \\
\hline
\end{tabular}

Mean \pm SD. ${ }^{* *} \mathrm{p}<0.01,{ }^{*} \mathrm{p}<0.05$. 
by the naturally occurring 16:1 trans (trans-palmitoelaidic acid) [10]. Other researchers also showed that palmitoelaidic acid is cardioprotective [11] [12]. As to relationship between IP-TFA or SFA intakes and CHD mortality, excessive intakes of both had a greater impact on risk for CHD in the US compared with Japan, whereas insufficient intakes of n6 PUFAs had about the same impact on risk in both countries [13].

As stated above, naturally occurring trans fats are consumed in smaller amounts (about 0.5 percent of total energy intake) in meats and dairy products from cows, sheep, and other ruminants; these trans fats are produced by the action of bacteria in the ruminant stomach [1].

Since trans fatty acids are not used in foods in Japan, all the trans fatty acids must come from meat or dairy products.

We found that there was no relationship between various foods intakes and plasma levels of trans fatty acids in Japanese old men. Only intakes of preference drinks such as tea and coffee had significant relationship with plasma levels of palmitoelaidic acid and linoelaidic acid.

These results seem to indicate that plasma levels of trans fatty acids are not derived from foods but derived by intestinal microbes.

There are some reports indicating that palmitoelaidic acid is a cardioproective factor [10] [11] [12].

The present reports may indicate that plasma trans fatty acids may be derived not by foods but by intestinal microbes. Such trans fatty acids are beneficial for health due to increase in a cardioprotective trans fatty acid such as palmitoelaidic acid.

The limitations of the present works were that the number of participants was not large enough. We try to continue the work by examining foods intakes and plasma levels of fatty acids. Also we should examine foods intakes and plasma FA levels of young people and old women in the future.

\section{Statistics}

The results are presented as means \pm SD. Spearman's correlation tests were used to examine statistical significance.

\section{Acknowledgements}

Experiments were designed and performed by all of the authors. AT wrote a manuscript. Statistical analyses were done by FS. All authors read the manuscript and approved the final version. All the authors had responsibilities for the final content. No conflicts of interest for any author.

\section{Ethics}

This work has been approved by the Ethical committees of Showa Women's University and Saiseikai Shibuya Satellite Clinic and has been carried out in accordance with The Code of Ethics of the World Medical Association (Declara- 
tion of Helsinki) for experiments.

\section{Financial Support}

This study was supported by grants by NPO "International Projects on Food and Health".

\section{References}

[1] Mozaffarian, D., Katan, M.B., Ascherio, A., Stampfer, M.J. and Willett, W.C. (2006) Trans Fatty Acids and Cardiovascular Disease. New England Journal of Medicine, 354, 1601-1613. https://doi.org/10.1056/NEJMra054035

[2] Mozaffarian, D. and Willett, W.C. (2007) Trans Fatty Acids and Cardiovascular Risk: A Unique Cardiometabolic Imprint? Current Atherosclerosis Reports, 9, 486-493. https://doi.org/10.1007/s11883-007-0065-9

[3] Mozaffarian, D., Micha, R. and Wallace, S. (2010) Effects on Coronary Heart Disease of Increasing Polyunsaturated Fat in Place of Saturated Fat: A Systematic Review and Meta-Analysis of Randomized Controlled Trials. PLoS Medicine, 7, e1000252. https://doi.org/10.1371/journal.pmed.1000252

[4] Jakobsen, M.U., O’Reilly, E.J., Heitmann, B.L., Pereira, M.A., Balter, K., Fraser, G.E., et al. (2009) Major Types of Dietary Fat and Risk of Coronary Heart Disease: A Pooled Analysis of 11 Cohort Studies. The American Journal of Clinical Nutrition, 89, 1425-1432. https://doi.org/10.3945/ajcn.2008.27124

[5] Farvid, M.S., Ding, M., Pan, A., Sun, Q., Chiuve, S.E., Steffen, L.M., et al. (2014) Dietary Linoleic Acid and Risk of Coronary Heart Disease: A Systematic Review and Meta-Analysis of Prospective Cohort Studies. Circulation, 130, 1568-1578. https://doi.org/10.1161/CIRCULATIONAHA.114.010236

[6] Stender, S., Astrup, A. and Dyerberg, J. (2012) A Trans European Union Difference in the Decline in Trans Fatty Acids in Popular Foods: A Market Basket Investigation. BMJ Open, 2. https://doi.org/10.1136/bmjopen-2012-000859

[7] Chardigny, J.M., Destaillats, F., Malpuech-Brugère, C., Moulin, J., Bauman, D.E., Lock, A.L., et al. (2008) Do Trans Fatty Acids from Industrially Produced Sources and From Natural Sources Have the Same Effect on Cardiovascular Disease Risk Factors in Healthy Subjects? Results of the Trans Fatty Acids Collaboration (TRANSFACT) Study. The American Journal of Clinical Nutrition, 87, 558-566. https://doi.org/10.1093/ajcn/87.3.558

[8] Takada, A., Shimizu, F., Ishii, Y., Ogawa, M., Takao, T., Koba, S., et al. (2018) Plasma Fatty Acid Composition in Men over 50 in the USA and Japan. Food and Nutrition Sciences. (In Press)

[9] Lemaitre, R.N., King, I.B., Mozaffarian, D., Kuller, L.H., Tracy, R.P. and Siscovick, D.S. (2006) Plasma Phospholipid Trans Fatty Acids, Fatal Ischemic Heart Disease, and Sudden Cardiac Death in Older Adults: The Cardiovascular Health Study. Circulation, 114, 209-215. https://doi.org/10.1161/CIRCULATIONAHA.106.620336

[10] Kleber, M.E., Delgado, G.E., Lorkowski, S., März, W. and von Schacky, C. (2016) Trans-Fatty Acids and Mortality in Patients Referred for Coronary Angiography: The Ludwigshafen Risk and Cardiovascular Health Study. European Heart Journal, 37, 1072-1078. https://doi.org/10.1093/eurheartj/ehv446

[11] Borgeraas, H., Hertel, J.K., Seifert, R., Berge, R.K., Bohov, P., Ueland, P.M., et al. (2016) Serum Trans Fatty Acids, Asymmetric Dimethylarginine and Risk of Acute Myocardial Infarction and Mortality in Patients with Suspected Coronary Heart 
Disease: A Prospective Cohort Study. Lipids in Health and Disease, 27, 15-38.

[12] Li, H., Zhang, Q., Song, J., Wang, A., Zou, Y., Ding, L. and Wen, Y. (2017) Plasma Trans-Fatty Acids Levels and Mortality: A Cohort Study Based on 1999-2000 National Health and Nutrition Examination Survey (NHANES). Lipids in Health and Disease, 16, 176. https://doi.org/10.1186/s12944-017-0567-6

[13] Wang, Q., Afshin, A., Yakoob, M.Y., Singh, G.M., Rehm, C.D., Khatibzadeh, S., et al. (2016) Impact of Nonoptimal Intakes of Saturated, Polyunsaturated, and Trans Fat on Global Burdens of Coronary Heart Disease. Journal of the American Heart Association, 5. https://doi.org/10.1161/JAHA.115.002891 